\title{
We are the Greatest Showmen: Configuring a Framework for Project-Based Mobile Learning
}

\author{
Dan Richardson \\ Open Lab \\ Newcastle University, UK \\ d.richardson@newcastle.ac.uk
}

\author{
Ahmed Kharrufa \\ Open Lab \\ Newcastle University, UK \\ ahmed.kharrufa@newcastle.ac.uk
}

\begin{abstract}
Little research has explored how mobile-learning technologies could be used by students to produce interactive artefacts during project-based learning processes. Following a design-based approach, we report on engagements spanning classroom and outdoor learning with students (ages 6-13) and teachers from three different UK schools and a summer school of Travelling Showchildren. Working within the time constraints of each context, we deployed a variety of configurations of a project-based mobile learning (PBML) framework intended to support the production of student-designed mobilelearning activities. We contribute insights gained from these engagements, including how mobile technologies can harness students' existing desire for independence and how they can be configured to leverage the physical and social attributes of place and community as learning resources. We argue for further exploration of the potential roles for mobile technologies within project-based learning, and contribute our PBML framework with recommendations for its re-configuration in response to contextual constraints.
\end{abstract}

\section{Author Keywords \\ Mobile-Learning; Project-Based Learning; Digital Civics}

\section{CCS Concepts}

-Human-centered computing $\rightarrow$ Empirical studies in interaction design; •Applied computing $\rightarrow$ E-learning;

\section{INTRODUCTION}

Blumenfeld et al. lament that small, easily assessed tasks which focus on low-level facts and skills (i.e. tasks commonly found on worksheets) have become the focus of many classrooms [5]. They argue that these tasks afford students ' $\mathrm{few}$ opportunities to represent knowledge in a variety of ways, pose and solve real problems, or use their knowledge to create artefacts (shareable and critiquable externalizations of students' cognitive work)'. This can be partly attributed to pressures and limitations put upon teachers and schools, as they work within structures which expect them to conform to quantifiable testing

\footnotetext{
Permission to make digital or hard copies of part or all of this work for personal or classroom use is granted without fee provided that copies are not made or distributed for profit or commercial advantage and that copies bear this notice and the full citation on the first page. Copyrights for third-party components of this work must be honored. For all other uses, contact the owner/author(s).

CHI'20, April 25-30, 2020, Honolulu, HI, USA

(C) 2020 Copyright held by the owner/author(s)

ACM ISBN 978-1-4503-6708-0/20/04.

DOI: https : //doi .org/10.1145/3313831.3376650
}

methods-propagating worksheet-style tasks and 'teaching to the test'. The head of the UK's Office for Standards in Education (Ofsted) has noted that '[Ofsted] have created a situation where second-guessing the test can trump the pursuit of real, deep knowledge and understanding' [20]. Blumenfeld et al. argue that a preferable alternative is project-based learning (PBL), which they describe as an approach to teaching and learning which focuses on engaging students through the investigation of non-trivial, 'authentic' problems in a manner which supports learner autonomy over the course of an extended project [5]. These projects frequently involve the creation of public artefacts, employing constructionist learning processes $[21,12]$. However, the restrictions placed upon teachers frequently impacts their available teaching time, curriculum content and pedagogical approaches. This has meant that implementing project-based learning in UK schools has proven to be a challenge [33].

At the same time, mobile learning ('learning across multiple contexts, through social and content interactions using personal electronic devices' [10], AKA 'm-learning') has grown to play a large role within schools in the UK, with access to tablet computers becoming more common in schools (44\% of UK schools are expected to have one tablet per child by 2020 [7]), and their general ubiquity meaning most of the younger population is familiar with their use (84\% of UK children aged between $8-16$ report owning a smartphone [32]). Despite this, there is a lack of HCI research around how m-learning technologies can be used for artefact creation throughout the PBL process [8].

Our research aims to explore how m-learning technologies could be effectively utilised within project-based learning processes in schools, particularly in students producing their own interactive learning resources. This paper explores the concept of 'project-based mobile learning' (PBML) through the creation of a PBML framework, and contribute insights from its application in studies held with three different UK schools and a summer school of Travelling Showmen. We contribute the framework, suggestions for its configuration in response to contextual challenges, reflections on how PBML harnessed students' existing desires for independence, and how it could offer new avenues for leveraging place as a learning resource. We conclude by arguing for further exploration of how mobile technologies can support the creation and sharing of interactive content within PBL. 


\section{RELATED WORK}

This section briefly introduces and discusses the purported benefits of constructionism and project-based learning, and how m-learning technologies can fit into the PBL process.

\section{Constructionism \& Project-Based Learning}

The learning theory of constructionism argues that constructing, sharing and reflecting upon 'public entities' (physical, virtual or even conceptual artefacts) can be a powerful way for learners to build 'knowledge structures' - collections of knowledge, concepts and facts interrelated through various semantic relationships [21]. Papert argues that the process of learning is the building of these knowledge structures, and is more likely to occur in contexts where the learner engages in the construction of public entities. Noss and Hoyles argue that constructionist working environments afford learners to take ownership of their learning approach, leading to indicators of greater engagement, confidence and empowerment [19]. They also posit that through exploration and construction of public entities, learners can encounter 'concepts and strategies that confront and build upon intuitive knowledge'. For this reason, they argue that constructionist tools need to be expressive enough to facilitate these concepts emerging through the learner's construction of public entities.

Project-based learning is an instructional pedagogy which presents learners with a given 'problem' or task, requiring them to investigate and work on a given subject over a longer period of time. These problems are non-trivial and often framed as 'authentic', in that they are somewhat applicable to the real world [5]. Frequently, students' projects will result in the creation of an artefact in response to a given problem (such as videos, reports, artworks, websites or performances [12]), in effect making PBL a method of applying constructionism in response to real-world problems and supporting the inclusion of prior knowledge, domain research and greater levels of student autonomy. Applications of PBL (and parallel pedagogies such as problem-based learning) conform to the same essential elements: a challenging problem or question; sustained inquiry; an element of authenticity; a degree of student control; reflection; critique and revision; and a final public product [16]. Previous research has argued that these projects can serve to build bridges between classroom activities and real-life experiences [5], enhance applied and conceptual knowledge around a subject [6], and that greater levels of autonomy and challenge can result in indicators of higher levels of student engagement [36].

While some studies have found that project-based instruction is not necessarily more demanding in terms of teaching time and resources [2], Blumenfeld et al. posit that by their nature PBL requires student engagement over extended periods of time [5]. Krajcik et al. argue that constructing knowledge in meaningful and situated activities can take students more time, leading to teachers being hesitant to put it into practice when faced with strict and competing curriculum goals [14]. The non-profit organisation Innovation Unit suggest that while it can be a powerful learning strategy, putting PBL into practice requires substantial changes in how teachers approach classroom structures, activities and tasks [13]. This is easier said than done, as governmental pressures and restrictions often limit the amount of time teachers can dedicate to particular topics and experiential learning methodologies which don't target given examinations (particularly in later school years, which place greater emphasis on quantifiable assessment) [20].

Project-based learning is recognised as a fertile ground for technology-enhanced learning. Bell argues that as long as it doesn't become the learning focus, technology can benefit all aspects of PBL (including research and data collection, knowledge sharing and artefact creation), and that tapping into students' existing computer fluency can boost engagement [4]. ChanLin describes how students used digital technologies within PBL for researching on the web, taking photographs, participating in online communities and creating web pages as final artefacts [9]. Heslop et al. found that the creation and sharing of interactive digital artefacts supported metacognitive skills, such as writing for an audience [11]. Sarangapani et al. explored how students could create interactive digital content as public entities to be shared with peers in other cultures, and argued that creating and sharing artefacts encouraged students to more deeply engage with the content [28]. However, these studies are of limited use for PBML, as they are either labbased [11] or do not use mobile technologies for the creation of interactive content $[28,9]$.

\section{Mobile Learning}

The adoption of mobile devices into UK classrooms has been dramatic, with nearly half of UK schools being expected to have one tablet per child within the next few years [7]. Mobile devices have been touted as having a number of advantages over their more stationary counterparts: for example, Traxler argues that mobile technologies can offer structured educational experiences which can be situated in-and responsive to-authentic learning environments [34]. This makes m-learning well situated to benefit from Lave and Wenger's Situated Learning Theory, which posits that learning occurs unintentionally in authentic activities and contexts through 'legitimate participation' in communities of practice [17]. Sharples et al. argue that to better utilise these advantages, m-learning technologies should take into account the physical and social aspects of the learning context, the amount of control the learner has over the activity, and the learner's communication with others [30].

Previous m-learning research has used these capabilities for a wide variety of applications, such as sensing tool kits to conduct citizen science [29]; enabling seamless learning across classrooms and museums on school trips [35]; and empowering children in collecting evidence to support their advocacy and engagement in urban design processes [22]. However, these projects focus on the consumption of knowledge or the collection of data, rather than the construction of content. Prior research has also shown that m-learning technologies can enhance the development of relationships with place, and act as a medium through which stakeholders can harness the underlying socioeconomic infrastructures of place as learning resources [25]. In the ParkLearn project, Richardson et al. attempted to combine elements of all of the above into a single mobile application supporting creating, sharing and 
engaging with bespoke, place-based interactive m-learning activities, which leverage both the learning environment and mobile hardware to support authentic situated m-learning [26]. Through using ParkLearn in longitudinal studies with schools and park volunteers, the authors found that the application promoted a sense of ownership in both learners and activity creators by supporting greater degrees of creativity and independence. The application was also shown to be an effective medium through which physical and social aspects of the local environment could be leveraged as learning resources. However, in these studies the ParkLearn activities were designed by teachers and community experts, and so were not part of a PBL process.

Chan et al. note that the explicit use of mobile technologies in PBL has been under-researched, but noted that students use mobile devices in multiple stages of the PBL process, including researching on the Internet, making notes, sharing materials and making use of applications to help understand abstract concepts [8]. Massey et al. argue that the creation of mobile technologies as final public entities can reframe students as developers and decision makers of mobile applications, rather than simply end-users [18, 23]. Sarangapani et al. held studies in which students used mobile devices to create video recordings for cross-cultural PBL, noting that the schools claimed the resulting videos were accessible and engaging learning resources [27]. Projects such as Science Everywhere demonstrate that sharing students' artefacts as public entities on social platforms can foster an element of ownership and appreciation of community resources [1]. Furthermore, multiple projects utilise m-learning for constructionist activities. For example, the Zydeco application supports inquiry-based, seamless learning between classroom and museum contexts by supporting the preparation of questions, and the creation of photos and tags in response to them [15]. While the authors do not position the app as a PBL tool (project creation is framed as being teacher-led), students could feasibly research and create their own Zydeco projects for their peers as public entities. Studies such as these suggest that m-learning technologies can be configured to support the PBL process, or even be used as platforms through which final public entities could be created. However, there exists little field-tested, generalisable guidance on how m-learning technologies could be used in this way within complex real-world PBL contexts.

\section{A PROJECT-BASED MOBILE LEARNING FRAMEWORK}

This project was undertaken through a design-based research (DBR) approach: one in which researchers work alongside practitioners (i.e. teachers) in naturalistic settings, and produce multiple iterations of designed interventions to explore theoretical relationships [3]. For these studies, we wished to explore the potential for m-learning applications to be used as constructionist tools within a PBL process, used by students to create new learning resources for use by their peers. To this end, we developed a PBML framework for use within UK schools. This final design was created iteratively, as a result of the findings covered in the Studies section. In this section we introduce the framework, and the OurPlace platform with which we chose to apply it.

\begin{tabular}{|c|c|}
\hline Task Type & Interaction Description \\
\hline Information & $\begin{array}{l}\text { Read some written information, with an optional } \\
\text { accompanying image and hyperlink }\end{array}$ \\
\hline Listen to Audio & Listen to a given audio recording \\
\hline Take a Photo & Use the camera to take still images \\
\hline Photo Match & $\begin{array}{l}\text { Use the camera to match an existing photo given } \\
\text { as an overlay }\end{array}$ \\
\hline Draw a Picture & Draw a picture onto a blank canvas \\
\hline Draw on Photo & Draw on top of a given image \\
\hline Record Video & Record a video using the camera \\
\hline Record Audio & Record audio using the device's microphone \\
\hline Map Marking & $\begin{array}{l}\text { Mark a given number of locations onto a Google } \\
\text { Map }\end{array}$ \\
\hline Location Hunt & $\begin{array}{l}\text { Track down a target location by observing your } \\
\text { reported distance }\end{array}$ \\
\hline Scan QR Code & Find and scan the correct $\mathrm{QR}$ code \\
\hline Multiple Choice & Choose a response from text options \\
\hline Text Entry & Enter a response using the keyboard \\
\hline
\end{tabular}

Table 1. The Task Types available in the OurPlace application

\section{The OurPlace Platform}

To capitalise on the advantages of m-learning technologies identified by Sharples et al. [30], we decided that we would use the framework with a situated learning approach, leveraging each school's surrounding heritage as a resource for educational content creation. As the ParkLearn application is available for free and was reported as being well suited to this [26], we hypothesised that using it to construct digital activities regarding local heritage would produce suitable final public entities for a project. OurPlace is the current iteration of the ParkLearn platform, with an expanded feature set and re-branded to encourage its use in contexts outside of local parks [24]. Consisting of a website and mobile applications for both Android and iOS, OurPlace supports the creation of - and sharing and engagement with-interactive $\mathrm{m}$ learning activities ('Activities'), each of which is built up from smaller, modular tasks ('Tasks'). These Tasks each consist of a specific interaction ('Task Type'), which either promote creativity, emulate traditional classroom learning materials or use the device's hardware to give context-specific experiences (Table 1). OurPlace also supports 'Follow-Up Tasks' that enable activity creators to add sub-tasks which unlock once their parent task has been completed (Figure 1.E). This supports the design of more complicated combinations of interactions (for example: a Location Hunt could unlock a Record Audio and Take a Photo once the user arrives at a designated location).

Activities are created within the app itself. After supplying a title, description and an optional image (Figure 1.A), the designer selects and configures the Tasks that make up the Activity (Figure 1.B). Each Task Type requires at least a written instruction for the learner, but some can require some additional customization (e.g. supplying coordinates on a Google Maps view) (Figure 1.C). No additional equipment or software is required to create activities (other than Scan the $Q R$ Code, which requires printing the QR code from the OurPlace website). Uploaded activities can be edited for testing, feedback and iteration - allowing for the 'essential elements' of reflection, critique and revision of a final public product [16]. 


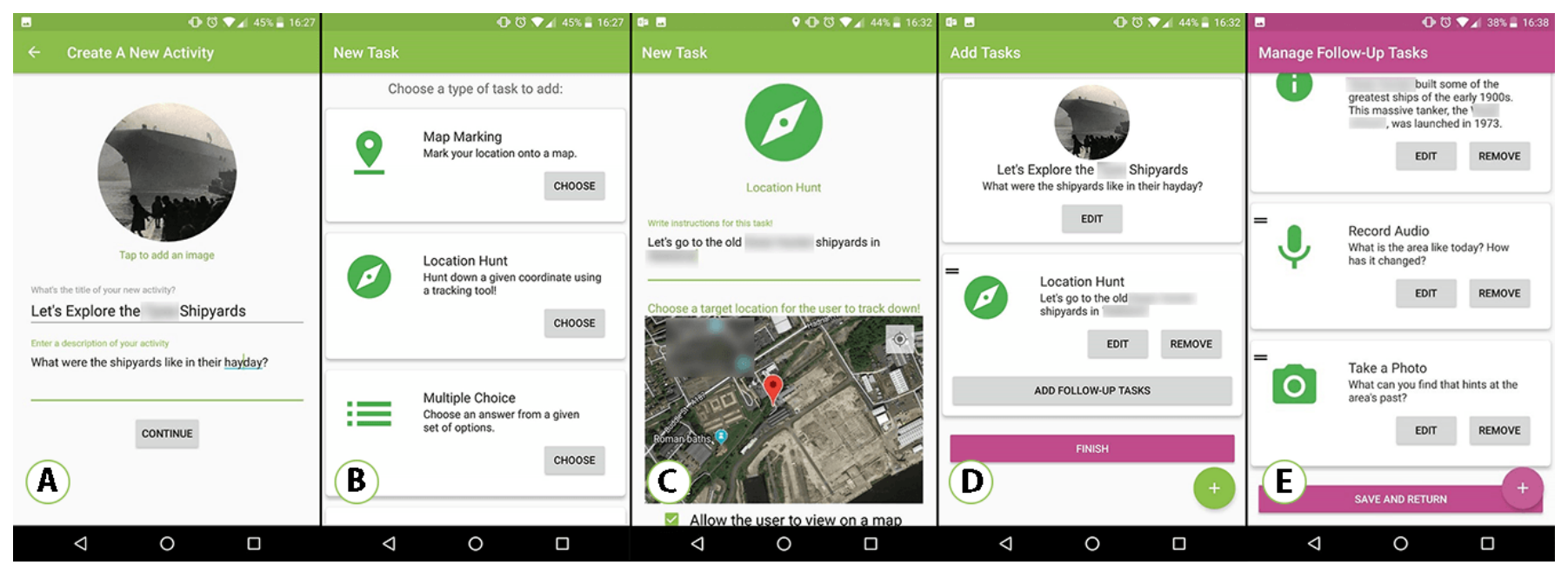

Figure 1. Creating an OurPlace activity (left to right): A) Choosing the activity's title, description and image; B) Choosing a Task Type to add; C) Adding a Location Hunt Task, with description and target; D) The new Task in the Activity; E) Adding Follow-Up Tasks to the Location Hunt

\section{Overview of the final PBML Framework}

We knew that any PBL programme would have to be able to adapt to variations in teachers' time, teaching requirements and levels of mobile hardware access [5, 14, 13, 33]. In response, we designed an adaptable five-stage framework based on the essential elements of PBL [16]. This framework asks students to create a mobile-learning artefact as a final public entity, following a series of PBL engagements in response to their teacher's chosen topic. The stages and our application of them with OurPlace are described below, along with suggestions for how other technologies could be used.

Demonstrating the Medium: This stage gives students a hands-on example of an exemplar application of the technology, allowing them to become familiar with its potential and encouraging them to bear the technology's capabilities in mind when formulating ideas during the following stages. For OurPlace, students should be introduced to the structure through completing an example Activity containing examples of how the different Task Types can be implemented. This would likely be similar with other technologies, unless they are simple enough to be self-explanatory.

Researching the Domain: As with most other forms of PBL, a significant amount of time is allotted to students investigating the given problem domain. This can be assisted by technology in the classroom (e.g. search engines) or even in-situ (e.g. using Zydeco to structure data collection during site visits, or while performing interviews). A degree of autonomy should be granted to the students, however some guidance (e.g. initial research topic) from a teacher may be required.

Prototyping: This stage was added after the first study, detailed below. In it, students create a low fidelity (e.g. pen and paper) prototype of their public entity, using their research as content. Our reasoning for doing this outside of the technology is that it: (i) doesn't require access to mobile hardware; (ii) lets students design without having to simultaneously learn the technology's authoring interface; (iii) emphasises the learning focus as being on the content, rather than the technology [4]; and (iv) supports visual learning and tangible interactions, thus more easily supports collaboration between students [31]. In the study by Sarangapani et al. [27], for example, students could prototype their videos by creating storyboards.

To prototype OurPlace Activities we created a jigsaw exercise, where students can design different configurations of their Activities (Figure 2). The jigsaw's structure is directly analogous to that of an Activity: a single piece is dedicated to the its title, description and cover image (i.e. Figure 1.A), with the Activity's Tasks represented as separate pieces connecting to it and chaining together. Each Task's jigsaw piece has a slot for a smaller Task Type piece, and the jigsaw allows for Task pieces to be connected in different directions to indicate Follow-Up Tasks. Pieces feature a layer of sticky-back plastic and are written on with dry-wipe pens, allowing the students to make amendments and the jigsaws to be reused. Students' finished prototypes are photographed for later reference.

Creating \& Refining: This stage involves the creation, testing and iteration of the public entity using the m-learning technology. If the students created a prototype, this could simply serve as a digitisation process. For OurPlace, this involves creating Activities within the mobile application, and requires guidance from the educator/researcher as to how the creation process works. Once completed, students may want to garner feedback, refining the artefacts in response to any issues encountered by their peers.

Sharing in the Wild: The final public entities should ideally be shared with peers or the wider community, as previous research has shown it can encourage students to engage more deeply in the creation stages by supporting critical thinking [28]. Students can exchange their uploaded OurPlace Activities with each other or communities outside of the school, and run them in authentic learning environments. Sharing OurPlace Activities goes beyond the traditional process, as the public entities themselves are interactive learning activities. Other technologies could be utilised to assist in sharing artefacts with other students or the wider community (e.g. using Science Everywhere to show and invite interaction with students' artefacts on public displays [1]). 


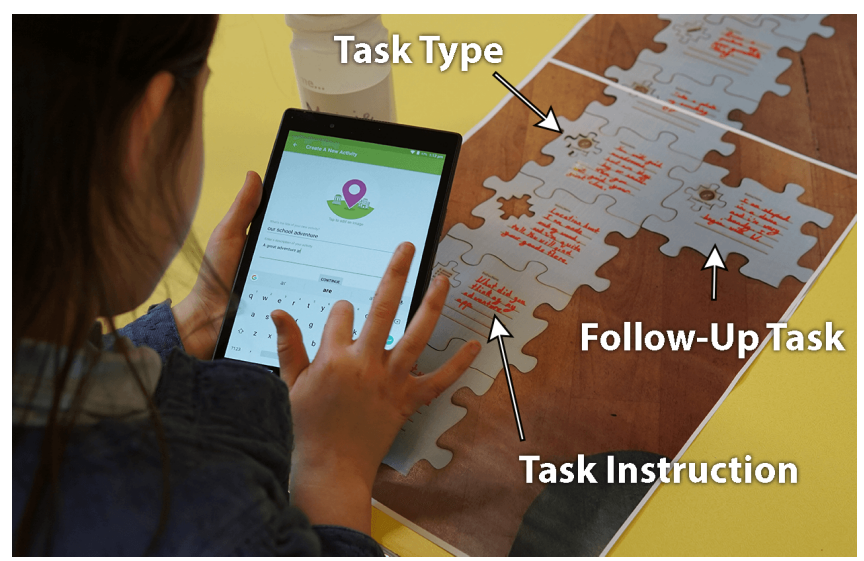

Figure 2. A student uses a photograph of their jigsaw prototype as a reference for creating an OurPlace Activity.

\section{STUDIES}

We developed this framework and assessed its performance within real school contexts by holding studies across the North of England, with three schools and a summer school of Travelling Showmen.

\section{Research Methods \& Data Collection}

Participant teachers were informed that we were investigating how to structure the use of mobile technologies and local community resources within school PBL activities. All of the engagements (class activities, teacher interviews) were audio recorded (totalling approximately 12 hours), the students' jigsaw prototypes (35 produced) photographed and OurPlace creations uploaded for analysis (72 Activities uploaded). A mix of semi-structured and unstructured interviews were held with the teachers after each engagement, with the aim of understanding how the sessions compare to their previous experiences and any ideas they had for the framework's development. Oral feedback was given by students during the engagements. Parental consent was acquired prior to each study, with additional consent requested for taking photographs. The recorded audio was reviewed, and any clear speech transcribed (outdoor recordings were sometimes unclear). The different sources of data (audio, photos, OurPlace data) were collated per engagement for inductive thematic analysis with exploratory, line-by-line coding. In-keeping with DBR methodology, this analysis was performed after the conclusion of each study, to better understand the performance of the PBML framework and how it could be improved. Most notably, this resulted in the introduction of the Prototyping stage, after it was found that the students in School 1 were concentrating more on the technology's novelty than the learning content. At the end of the project the codes were categorized into themes, which are presented for discussion later in this paper: Configuration and Compromise, Harnessing Students' Desire for Independence, and Leveraging Place through PBML. The anonymised findings reported in this section pertain to these themes.

\section{Configuration 1: Without Prototyping}

We first engaged with a Year 8 history class (age 12-13, N=32) in a secondary school (School 1) in a moderately affluent village. The school had been involved in previous projects with our research group. We approached its leadership about the possibility of doing a PBML project: the school's headteacher agreed and assigned the class's history teacher (T1) to the study. As with many other secondary school teachers in the UK, T1 was under pressure to prepare the students for frequent formal assessments, and so was reluctant to dedicate much teaching time to the project. When we were organising the study, they noted: 'Workload and time would be the main issues. It would be difficult to slot something additional like this in around key assessment work, and also keeping it relevant to the curriculum we are following'. As a result, the project was given a very short amount of classroom time-only two hour-long sessions, with further work to be done by students outside of school. Observations of two of T1's other lessons suggested that they preferred an 'authority' or lecture style of teaching. T1 appeared ambivalent towards PBL approaches, and when queried on their opinion of them noted 'It's not the way we do things here'.

T1 already possessed a paper-based history trail of the local village, designed for use by younger students joining the school. For the study, T1 tasked the class to use OurPlace to create digital trails in pairs, featuring historical elements of their choosing. Prior to our first session with the students, T1 provided them with a lengthy PDF document and a PowerPoint presentation, which detailed most of the historical buildings in the village, serving a starting point for researching content. Prior to the first session, T1 expected that the students should be well prepared in terms of trail content: 'The students should already have ideas of what they want to do. The issue is what the software can do and whether it can easily tied in with the plans they have made already.' As such, the 'Researching the Domain' stage took place earlier in the process than planned. T1 was also concerned about the students' work being able to function outside of an m-learning context, hoping to also have 'analogue' versions of the students' trails: 'Could a finished product be adapted to be used at another time even if no iPads were available - maybe some of the ideas usable in a non-digital way?' To this end, they encouraged students to try and design their digital trails such that they would be adaptable to a pen and paper format.

T1 decided to make the majority of the Activity creation process a homework task, with students using their own devices outside of school. This was mainly in response to the extremely limited amount of classroom teaching time that could be dedicated to the project: because we also wanted to go through the students' final Activities, that only left a single hour-long session to work with the students in class. In order to prepare the students for this independent work, our first hour-long session was spent Demonstrating the Medium. This involved the students completing an example OurPlace Activity on the school grounds, demonstrating all of the Task Types and several instances of Follow-Up Tasks. Upon returning to the classroom, the rest of the session (around 20 minutes) was spent introducing the students to OurPlace's Activity creation tools. By the end of the hour, all students reported that they understood the app and how to make their own Activity.

During the second session three weeks later, the researcher and T1 sat with the pairs of students in turn. This served both 
as a way to examine the students' creations, and to gain verbal feedback from them about their experiences. While most of the pairs had produced an Activity, they tended to be quite short (averaging 4 Tasks per Activity) and shallow: most of the Activities served more as explorations of the different interactions possible with OurPlace than a meaningful engagement with the subject matter. For example, one student's Activity asked the learner to simply find and photograph an 'area of interest'. Despite this, many of the students had engaged strongly with the process and had taken ownership over their Activities: for example, one student's trail featured characters they had created for their personal YouTube channel. However, as a result of the focus on technology interactions, many students struggled in fulfilling the teacher's requirement of creating a paper-based version of their Activity. One pair of students had more success, claiming 'I think we've found it easier than other groups because we focused more on the content than using all of the different interactions. So a lot of the content can be the same, it's just changing how to interact with it'. Due to teaching time limitations, the Activities were not shared between students or used outside of the classroom.

T1 noticed the students' focus on interactions with the technology over engaging more deeply with the historical content. In the follow-up interview, they noted: 'The thing I kept coming across was the lack of challenge, the lack of depth. Really just playing with the technology rather than [engaging with the history]'. This surprised T1, as they had been expecting any issues encountered to have resulted from the introduction of new technology, rather than the students' research: 'I think it's more of a success for the medium than the actual content. [...] Maybe not what I expected, actually-in some ways maybe the opposite'. T1 argued that without the deeper integration of research and knowledge into the Activities, they are of little value: 'It needs to be worth doing: there's no point in having all of the bells and whistles if there's no substance'. They noted that this could have been improved through a reconfiguration of the PBML framework to offer more structure: 'It's worth cogitating about what parameters you probably need to introduce, to guide them towards deeper thinking. I know that if that had been more free-form and open-ended, that would have been rather worse.' After considering these findings, we developed the Prototyping stage of the framework to assist in students giving their Activity's structure greater consideration.

\section{Configuration 2: Full Process}

We worked with two different schools over an extended period of time (10-12 hours of sessions per school, spread over several weeks) to test this new, longer implementation. This was partly supported by the fact that we were working with Year 4 (age 8-9) classes - we found that pre-secondary school teachers are more willing to engage with experiential forms of learning, as less focus is placed on examination prep. Both schools welcomed having longer projects over several sessions.

\section{Engagements with School 2}

The first of these schools (School 2) was based in a tiny rural village. We worked with the entirety of the Year 4 group, who were the oldest children in the school (age 8-9, N=7). Because the school's population is so small, Year 3 and Year 4 share the same classroom. The class teacher (T2) had approached us about using PBML to augment orientation and map-reading with new technologies in lessons, after attending one of our related workshops. With a generous scope and large amount of time available, we decided that the students would do two projects: one to learn the mechanics of making Activities, and another which focused on their village's heritage.

For the first project, we tasked the Year 4 students with individually creating OurPlace Activities for their younger classmates to complete around the school grounds. As with School 1, the first session started Demonstrating the Medium through an example Activity. We then moved straight to the Prototyping phase, as these first projects didn't require any research. While the students initially struggled conceptually with the jigsaws due to their abstract nature, after a few minutes they understood the links between the puzzle pieces and the structure of the app. The children all settled on creating some form of 'adventure', where each Task was a riddle to solve in order to find locations within the school. Tasks included finding QR codes around the school, and finding specific locations using 'Photo Match'. We found that their Activities made use of most of the different Task Types available, which may have been encouraged by the jigsaw packs having a limited quantity of each Task Type piece, forcing students to diversify (however, some students overcame this by simply not placing a Task Type piece if their chosen one wasn't available-see Figure 2 as an example). This highlights the potential for prototyping in a physical medium to provide constraints.

In the second two-hour session the students used their jigsaws as references to assemble their OurPlace Activities (Figure 2). Most were happy working independently, and reported that their jigsaws made learning the creation process 'much easier'. The children enjoyed exploring what they could do with the technology's functionality: for example, one student's final Listen to Audio Task 'rewarded' the user for completing their Activity with a recording of them singing the song Celebration by Kool \& the Gang. The second session concluded with the students testing out their Activities and making refinements (mostly involving spelling errors and reordering Tasks).

The final two-hour session of the project was spent by the students sharing with their peers. T2 briefed the younger students, giving the Year $4 \mathrm{~s}$ positions of seniority and highlighting their efforts: 'You really need to listen to what [Year 4] have to say, because they have designed this themselves. They are your teacher, OK? Please listen, because they've worked really hard, and they're really excited about you having a go.' The Year $4 \mathrm{~s}$ accompanied rotations of small groups of younger students as they completed their Activities around the school, with groups being swapped to allow all students to try each Activity. The Year $4 \mathrm{~s}$ were given the responsibility of showing the younger students how the app worked, and assisting them if they got stuck. The younger students were very enthusiastic, and were keen on making sure they completed each of the Year $4 \mathrm{~s}$ 'Activities. At the end of the session the Year $4 \mathrm{~s}$ hosted a school assembly, in which they showed the other children their jigsaws and shared what they most enjoyed ('I enjoyed being the teacher'; 'Being outside'; 'I enjoyed making the 
Activity itself') and the younger children gave them feedback ('Our favourite was [Susan's], because we got to find lots of things'; 'I really liked the beeping one, the Location Hunt'). The school's headteacher praised the Year 4s' independent work as showing maturity: 'We can trust you to do something away from the class teacher and still do something really good. I think you really are stepping up to be Year 4s, it's wonderful to see. [...] If you're very grown up, you get to do very grown up things. So let's give Year 4 a clap'. T2 also praised their leadership ('I would like to also point out how good they were as teachers, as well. They really came into their own. I was very proud of them.') and noted that OurPlace supported a varied output: 'They were very different as well weren't they? The ideas. Even though you all started off with the same tools.'

Following the success of the first project, T2 was excited to start the second one. Prior to it starting, T2 preemptively collected a number of historical resources relating to the area, including newspaper articles, photographs and a book detailing the village's buildings. As T2 claimed to not have much prior local historical knowledge, this also served as their introduction to the village's heritage. T2 proceeded to make a shortlist of the more interesting buildings (such as an old blacksmith, a pub and a post office), shared these with the Year 4 students, and then took them on a short walking trip around the village so that all of the children had first-hand experience with the sites. Each child chose a different location to base an Activity on, using the walk and the teacher's resources to research it.

As the group was already familiar with the app, we skipped the Demonstrating the Medium stage. When we asked the students if they would find it helpful to plan their Activities out using the jigsaws, they said no: they'd rather jump straight into making them using OurPlace, as they were already comfortable creating and editing using the app's tools. The Activities were produced over three hours between two sessions. All of the children's Activities featured 'Location Hunt' Tasks guiding the learner to their chosen location, with further details delivered through 'Information' and 'Listen to Audio' Tasks. Less passive Tasks included asking the learner to draw details from the location, and a 'Multiple Choice' quiz based off given information. The students then shared their Activities with the younger students in three groups, with each group accompanied by an adult and sharing tablets one-between-two. The groups completed each of the 7 Activities around the village before returning back to the school, where the researcher asked them for verbal feedback. T2 was pleased with the Activities, as the students 'were able to engage with the local history in a way which they enjoyed, and they've taken pride in sharing their work with the Year 3s.' After the trip, several of the younger students asked if they could make their own Activities the following year.

\section{Engagements with School 3}

Newcastle City Council's Urban Design and Conservation Team connected us with a Year 4 teacher (T3) at an inner-city school (School 3), as their class had shown an interest in local history by campaigning for the installation of a commemorative plaque for a notable slavery abolitionist who had lived nearby. This was particularly relevant to School 3, as it serves a large number of families of Nigerian descent. T3 was enthusiastic about the concept of producing Activities relating the the area's numerous other plaques: 'I' $m$ very interested as it had occurred to us that the plaques make a great learning resource on our doorstep'. We worked with the majority of the school's Year 4 (age 8-9, N=21, led by T3) and Year 6 (age 10-11, N=32, led by Teacher 4) students, who worked together on the project in 14 mixed groups of 3-4.

As with School 1 and School 2, the students used a demonstration Activity as an introduction to OurPlace. Following this, T3 explained that each group was to choose and research one of the historical figures commemorated by the plaques in the area, and produce an Activity related to them. Over the following week, the teachers dedicated several hours of class time to researching and visiting the plaques. As a result, by the second engagement each group had prepared several pages of notes relating to their chosen plaque and were ready to start designing their Activities. The children found it helpful to have a tablet for reference while constructing their prototypes, and frequently referred to their notes while writing their Tasks. The completed jigsaws were photographed and used as references for creating the Activities in a third session later that week. Part of this third session was also spent visiting the plaques with the students, so that they could test and refine their Activities and take photographs to include in them. The final session of the project involved the students going out and completing each other's Activities. Examples of the final Tasks include asking learners to 'Record Video' of interviews where students role-play as their plaque's person of interest, and Follow-Up Tasks quizzing learners about the contents of 'Listen to Audio' narrations. Some Activities lacked content, which T3 attributed to a lack of available information for their plaque's subject and some children's behavioural issues.

Verbal feedback was requested from the students once back in the classroom. Several mentioned that they particularly enjoyed sharing Activities: '[I most enjoyed] today, getting to go around and swap with other people and getting to find out about theirs.' They also felt that swapping Activities was an important part of the process, as it could also share different ways in which the Task Types could be used. Furthermore, one Year 6 student noted that OurPlace could be used to share the value of place with visitors and other communities: 'I think that if we made stuff for another school that made them learn, it would be really good because you could make it about your school.' Teacher 4 expanded on this concept: 'That would be an interesting thing to do, wouldn't it? To swap it and see what their daily life is like and what yours is like.'

During a follow-up interview, T3 reported to particularly like tactile nature of the jigsaw prototype: 'Doing it on a piece of paper is very boring, so to get them to understand that the order can matter... I think that's a very good, visual way of showing the children. That really worked.' Unlike the children in School 2, T3 also saw value in doing the prototyping for making further Activities: 'I would use the jigsaws every time. Because it's a different Activity.' T3 saw the jigsaws as Activity prototypes, rather than simply a way of easing the 
students into the application's structure. Following the study T3 requested a digital version, so that they could print copies for students to prepare future OurPlace Activities. T3 also saw a potential value in exchanging with separate groups of students: 'I think it would have been better if we'd done it, and then taken a different group of children out to use it. So you do it with one class, then take the other class with them to show it. And then they can evaluate by watching the other child. But it's just time pressure, isn't it?'

T3 was also highly favourable of teaching in authentic contexts and using the children's existing relationships with place: 'It was all about taking a context specific approach, that's what I'm really into. These children know about their local area, and that helped us scaffold the Activities.' T3 also noted that many of the children were not aware of the area's history, and that the historical figures could act as inspirational role models: 'This is where the children live, so it's really important that they understand the history of it. Really great people who're like them have lived in this area.' Furthermore, working in these environments brought the children in contact with community stakeholders: 'They got to meet people when they went out and about. They met the guy who's raising money for the sculpture in the middle of the park.' T3 also argued that using constructionist m-learning in a PBL approach helped leverage these civic resources in lessons, as the creation of public entities acted as a motivating factor: 'It was how we were going to bring those [resources] into our lesson. I think that OurPlace really helped: it gave us a focus to do the history through the app, rather than just go and collect the information and then-what do we do with it?'

\section{Configuration 3: Without Demonstrating the Medium}

Through discussions with T3, we discovered that they lead a summer school for children of families who run the local annual funfair. Following our engagements with School 3, we were invited to run a short engagement with a group of children (age 6-9, N=16) attending the summer school. These were the children of Travelling Showmen and Showomen, members of the Showmen's Guild: a trade association made up of traditionally insular cultural groups of families, who travel around the UK to run funfairs and circuses. While the children of these families (Showchildren) are registered with traditional schools, the families travel so frequently (one family claimed to work 40 events a year) that their schools send out packs of educational materials for the children to work on remotely. T3 derided these worksheet-based packs as uninteresting: '[The school packs] are super boring and often rubbish. Some schools are alright, but it's still working from a piece of paper.' Inspired by Teacher 4's suggestion that OurPlace could be used as a medium through which daily life experiences could be shared, T3 suggested that we do a short project at the summer school: 'Their lives are so different, it would actually be a nice tool to share with other children what it's like to be a Showman.' We arranged a trip for the Year 4 class from School 3 to visit the fair, where the Showchildren would introduce them to their ways of life. The Showchildren would create OurPlace Activities, with which Year 4 could also collect data for later classroom use.
As the summer school only ran for two weeks, we only had one three-hour session in the summer school in which to introduce the Showchildren to OurPlace and have them create Activities. Following the issues found in the configuration used in School 1, we decided to try another, skipping Demonstrating the Medium and relying on verbal instruction during the new Prototyping stage as an introduction to the app's functionality. The Showchildren were able to complete the jigsaw activity without first needing to use the application, and they gravitated towards making Activities which focused on their families' rides and stalls within the fair (e.g. Information Tasks about their families, Video and Photo Tasks capturing the rides in action). Transitioning from the jigsaw prototypes to the digital versions went similarly to the previous engagements, suggesting that the jigsaw serves as an intuitive metaphor for the application. Due to time limitations, the Showchildren were unable to test and refine their Activities.

We then ran a session in School 3, to introduce Year 4 to the concept of Travelling Showmen. The class's discussions largely centred on the concept of the Showchildren working in the fair, an idea which appeal to them: one child noted they wanted 'to get money to support my family'. However, there was a concern that as children, they wouldn't be treated as equals by adults: 'you might not get paid as much, because people could want to only go to adults and think that children are not responsible yet'. The children found the idea of inherited careers generally unappealing (most Showmen families have an occupational lineage of several generations), saying ' $I$ don't want to do my parents' job', and 'it's natural to want to do something different, [...] if you just carried on a tradition you might not really like it.' To encourage fruitful conversation between the two groups of children, we also asked Year 4 to prepare some questions to ask the Showchildren. Many of the questions revolved around the Showchildren's independence and influence in the community (e.g. 'Have you ever designed a ride?'), their work-life balance ('Would you like shorter or longer shifts?') and their earnings ('How much money do you earn?'). Comparatively few of the questions focused on social aspects of the Showmen community (e.g. 'Do you have any relatives who are in a different part of the world?').

The trip occurred the following week. An education specialist from the Showmen's Guild gave a short talk to the children regarding Showman ways of life, and their experiences growing up within the community. Following this, the Showchildren and Year $4 \mathrm{~s}$ were put into mixed groups of 4-5. Each group were given two tablets with the Showchildren's Activities, and the Showchildren were asked to guide the visitors around the fair. The Year 4s also used the Showchildren's Activities for their data collection, using the app to catalogue photos of the different rides and using Record Audio and Record Video Tasks to capture the Showchildren's responses to their prepared questions. Most of the questions had shifted to being about the lived experience of the Showchildren ('In a year how many places do you think you travel to?', 'Do you get to make many friends outside of the fair?'). The Showchildren also responded with some questions of their own, querying the Year 4s' experiences with the funfair. One of the Showchildren was particularly excited for the students to complete a 
'Scan the QR Code' Task, which asked them to find the child's parents' ride. However, when it was scanned they were disappointed to find that it didn't unlock any content, as there were no Follow-Up Tasks set. When this was explained, they didn't know what Follow-Up Tasks were: 'What did you mean by follow-up? How do you put something in it?' While disappointed, the child expressed interest in downloading OurPlace to make Activities at home.

\section{DISCUSSION}

These studies have shown that configuring the PBML framework differently can meaningfully impact students' engagement with and knowledge of the domain and technology.

\section{Configuration and Compromise}

In these studies it was necessary to adapt our proposed framework in response to each teaching context's limitations. For example, T1 was severely limited in how much time they could dedicate due to obligations to follow a strict curriculum, and the Showmen's summer school was only running as long as the funfair was in town. It was only when working with the younger classes in Schools 2 and 3 that more time could be afforded. This was certainly not ideal for PBL-style engagements, which should engage students over the course of an extended period of time [5]. Nevertheless, we argue that teaching contexts are rarely ideal, and so approaches to working within them should be adaptable and open to compromise. We wanted to explore this by implementing the framework in various real contexts which necessitated its adaptation.

We believe that skipping the prototyping stage in School 1 contributed towards the lack of engagement with the domain and put the students' main focus on the technology itself. Before taking the project away as homework, the last engagement the students had with the project was the technology's demonstration, meaning it was likely to take centre-stage in their minds. Our observations at Schools 2 and 3 suggest that inclusion of the prototyping jigsaw exercise may have brought the School 1 students' focus back to the domain's content.

The studies also highlighted the value of students sharing their creations with their classmates and/or the wider community. Students from Schools 2 and 3 emphasized the exchanging of Activities as being a particular highlight of their experiences, with students keen to both see what their peers had produced and also show off their own creations. T3 noted that had time allowed, they would have extended this sharing stage to support peer feedback between classes and groups. For the School 1 students, the lack of emphasis on sharing the created Activities with their peers or outside communities may have also reduced their value as constructionist public entities [21].

In response to these findings, our other time-limited engagement with the Showmen's summer school used a different configuration which skipped Demonstrating the Medium, instead focusing on the Prototyping stage. While the jigsaws seemed to provide a somewhat serviceable introduction to the app due to the closeness of its metaphor, the full capabilities of the technology had evidently not been made clear to the Showchildren. In the case of the Showchild with the QR code, this resulted in a degree of frustration and disappointment that their Activity wasn't as fully featured as it could have been. It's unsurprising that more advanced functionality, such as Follow-Up Tasks, would be unclear to children without first demonstrating them in an example Activity.

Each configuration held its own compromises, resulting in an interesting balancing act between four elements: classroom time; supporting the learners' understanding of the technology (omission of the Demonstrating the Medium stage led to not using the its full potential); supporting the meaningful application of content knowledge to that medium (omission of the Prototyping stage led to shallow public entities which focused on the technology rather than the content); and enabling learners to exchange their creations, knowledge and feedback within authentic learning environments (omission of the Sharing stage reduced the value of the entity creation process). Each of these elements was shown to be important, and compromising on any of them while still producing successful PBML engagements was difficult. However, successive projects can mitigate this by omitting certain stages as the learners become more experienced. This was shown in School 2, where the students opted to skip repeating the Demonstrating the Medium and Prototyping stages. Even this was up to some degree of teaching interpretation, as T3 noted they would use the jigsaw prototypes each time their class made new Activities. Further workarounds and compromises could also be explored: for example, T1 opted to make the Creating $\&$ Refining stage a homework activity, saving on classroom time. However, this raises issues around remote support and ensuring equal access to technology resources (potentially highlighting economic disparity between students).

We suggest that researchers and practitioners should weigh-up these compromises when configuring the framework according to their own motivations. For example, if full utilisation of the technology's potential isn't a priority, then the impacts of omitting Demonstrating the Medium become less of a concern. Likewise, skipping the Prototyping stage might be acceptable if the use of the technology is one of the main learning goals. However, we argue that omitting the other stages should be avoided as they are either key to the PBML process (research, creation) or major motivating factors (sharing public entities).

\section{Harnessing Students' Desire for Independence}

In these studies, many of the students we engaged with had a great desire for independence and to be respected as individuals. This was particularly evident in the School 3 class's fascination with the Showchildren's contributions to their family businesses, their lamentation at their lack of perceived responsibility when compared to adults and a desire to walk their own path rather than simply emulate their parents.

The PBML process capitalised on this quality, granting the students greater control and autonomy over their work [19, $36]$, and enabling them to approach creating Activities with greater degrees of personal input [25]. This could be seen in some of the personal touches put into their Activities, such as the Year 4's rendition of Celebration, or the Year 8's usage of their YouTube characters. These flourishes-alongside the uniqueness of each Activity-suggest that OurPlace conforms to Noss and Hoyles' requirement that constructionist tools 
should be expressive enough to support exploration and ownership through construction [19]. This also echoes previous research held with its predecessor, ParkLearn, in which students' sense of ownership of their responses to others' Activities was an important contributing factor towards their enthusiasm, thanks to greater degrees of freedom and independence [26]. Furthermore, the students' investment in their projects supports arguments that the greater autonomy afforded by PBL, as well as tapping into students' fluency with technology, can result in indicators of greater student engagement $[36,4]$.

When sharing their Activities with their peers, the students were clearly proud of their creations and enjoyed taking a 'teaching role': they took care to guide the other students through the Activities to avoid them getting stuck without being overbearing (as shown in School 2). The School 2 teachers rewarded the students' performance by playing to their desire for perceived maturity, noting their growing trust in the children to perform independently and recognising their seniority amongst the students. This feedback also served as qualities for the younger students to aspire towards-some of the younger students later enquired about making their own Activities when they're older. This move to reposition the Year 4 students from a 'consumer' role to one of mentorship-in which they have a degree of authorship over teaching materialsmirrors the study by Massey et al, in which they re-framed students from end-users to software developers and decision makers [18]. In both cases, the learners were empowered through the creation of public entities to be able to take an active decision-making role in how technology could be used within their learning environment. The addition of the sharing stages took this a step further, granting the students the gratification of seeing others enjoy their creations.

We suggest that future m-learning designs can harness student's desires for independence as a motivational force by granting them opportunities for autonomy and personal flourishes, as well as platforms through which to share these personal creations. Within these studies, this was achieved through a combination of technology, configuration and context: OurPlace elevated students from consumers to producers by granting them creative control, and the creation and sharing of public entities in authentic contexts at the culmination of a PBML process bolstered their self-worth and empowered them through positions of mentorship. However, it's also worth noting this approach may not be conducive to success in all contexts, as teaching styles and cultures within different schools may be at odds with it or require re-balancing. For example, T1 was in favour of greater scaffolding, and believed that further student autonomy would be detrimental.

\section{Leveraging Place through PBML}

Project-based m-learning offered new ways and motivations for the schools to engage with their area's local heritage and community, surfacing 'new' educational resources which had previously gone underused. For example, T2 reported to previously know very little of the village's local history, and School 3 hadn't previously made use of the commemorative plaques as learning resources. T3 argued that using OurPlace to create the public entities gave lessons a focus and motivation needed in engaging teaching sessions, as simply collecting information would have felt aimless. T3 was also ardently in favour of the students learning more about their local context, as the historical figures within the area could serve as inspirational role models. Finally, in the Showmen study, a (compressed) PBML process also supported exposure to and greater understanding of another community's heritage. The students' preconceptions regarding the Showchildren were corrected following technology-mediated personal interactions with the community, and their topics of interest shifted from the practical to the lived experience of being a Showman.

We argue that PBML is particularly well suited to leveraging these local resources, as it combines the advantages of project-based learning (supporting the ownership and exploration of ideas through the construction of public entities, in a process which encourages student inquiry and autonomy $[19,16])$ and those of situated, outdoor learning (experiential learning, embedded in authentic contexts [17]). M-learning technologies are uniquely suited to assist this process, due to their ability to leverage these authentic physical and social learning resources and support greater degrees of student control, communication and creativity seamlessly across different learning environments [30,26]. As noted by Chan et al., the use of mobile technologies in project-based learning has been under-researched [8]. Given these apparent advantages, we would like to see future research explore how PBML could benefit from other technologies which support the creation and sharing of interactive content, rather than simple knowledge consumption or the creation of passive artefacts (e.g. blogs).

\section{CONCLUSION}

We have reported on and discussed four studies exploring different configurations of a project-based, m-learning framework in distinct learning contexts. A limitation of this research is that they were only loosely tied into each school curriculum, and didn't assess learning outcomes. We hope that future engagements can offer deeper integration into existing curricula and feature formal assessments, supporting further insights into how PBML can fit into wider school systems. We gained an appreciation of the importance of understanding each learning context, and how configuring the use of technologies to fit the particular demands of a context can result in effects which compromise the learning experience. We also discussed how the PBML process can harness students' existing desires for independence as a motivational force by granting them opportunities for autonomy, creativity and personal flourishes. Finally, we discussed how PBML offered new avenues for schools to leverage their local heritage as learning resources, and argue that the potential roles for m-learning technologies within project-based learning processes should be further explored.

\section{ACKNOWLEDGMENTS}

This research was funded through the EPSRC CDT in Digital Civics (EP/L016176/1). Data supporting this publication is openly available under an 'Open Data Commons Open Database License' at: https://doi.org/10.25405/data.ncl.11534985. Please contact rdm@ncl.ac.uk for access instructions. 


\section{REFERENCES}

[1] June Ahn, Tamara Clegg, Jason Yip, Elizabeth Bonsignore, Daniel Pauw, Lautaro Cabrera, Kenna Hernly, Caroline Pitt, Kelly Mills, Arturo Salazar, and others. 2018. Science Everywhere: Designing public, tangible displays to connect youth learning across settings. In Proceedings of the 2018 CHI Conference on Human Factors in Computing Systems. ACM, 278.

[2] Sulaiman M. Al-Balushi and Shamsa S. Al-Aamri. 2014. The effect of environmental science projects on students environmental knowledge and science attitudes. International Research in Geographical and Environmental Education (2014). DOI : http://dx.doi.org/10.1080/10382046.2014.927167

[3] Sasha Barab and Kurt Squire. 2004. Design-Based Research: Putting a Stake in the Ground. Journal of the Learning Sciences 13, 1 (2004), 1-14. DOI : http://dx.doi.org/10.1207/s15327809j1s1301_1

[4] Stephanie Bell. 2010. Project-Based Learning for the 21st Century: Skills for the Future. The Clearing House: A Journal of Educational Strategies, Issues and Ideas (2010). DOI :

http://dx.doi.org/10.1080/00098650903505415

[5] Phyllis C. Blumenfeld, Elliot Soloway, Ronald W. Marx, Joseph S. Krajcik, Mark Guzdial, and Annemarie Palincsar. 1991. Motivating Project-Based Learning: Sustaining the Doing, Supporting the Learning. Educational Psychologist (1991). DOI : http://dx.doi.org/10.1080/00461520.1991.9653139

[6] Jo Boaler. 1999. Mathematics for the moment, or the millennium? Education Week (1999).

[7] British Educational Suppliers Association. 2015. Tablet adoption continues to rise; barriers to adoption shift BESA. (2015). https://www. besa.org. uk/news/besapress-release-tablet-adoption-continues-risebarriers-adoption-shift/

[8] Lap Ki Chan, Susan M Bridges, Iain Doherty, Manwa L. Ng, Jun Jin, Neel Sharma, Nam Kiu Chan, and Henrietta Yan Yu Lai. 2015. A Qualitative Study on How Health Professional Students and Their PBL Facilitators Perceive the Use of Mobile Devices During PBL. Interdisciplinary Journal of Problem-Based Learning (2015). DOI : http://dx.doi .org/10.7771/1541-5015.1510

[9] Lih Juan ChanLin. 2008. Technology integration applied to project-based learning in science. Innovations in Education and Teaching International (2008). DOI: http://dx.doi.org/10.1080/14703290701757450

[10] Helen Crompton. 2013. A historical overview of mobile learning: Toward learner-centered education. In Handbook of Mobile Learning. 3-14.

[11] Philip Heslop, Anne Preston, Anna Reid, and Ahmed Kharrufa. 2017. Studets' Perceptions of Learning Processes As Co-authors of Digital Tabletop Activities. In Proceedings of the 31st British Computer Society
Human Computer Interaction Conference (HCI '17). BCS Learning \& Development Ltd., Swindon, UK, Article 13, 5 pages. DOI:

http://dx.doi.org/10.14236/ewic/HCI2017.13

[12] Renata Holubova. 2008. Effective Teaching Methods-Project-based Learning in Physics. US-China Education Review (2008), 27-35.

[13] Innovation Unit. 2016. Why the UK needs to understand when and how PBL really works I Innovation Unit I New solutions for thriving societies. (2016).

https://www. innovationunit.org/thoughts/why-the-ukneeds-to-do-more-to-understand-when-and-how-projectbased-learning-really-works/

[14] Joseph S Krajcik and Phyllis C Blumenfeld. 2006. Project-Based Learning Handbook. DOI : http://dx.doi.org/10.1017/CB09780511816833.020

[15] Alex Kuhn, Clara Cahill, Chris Quintana, and Shannon Schmoll. 2011. Using tags to encourage reflection and annotation on data during nomadic inquiry. In Proceedings of the SIGCHI Conference on Human Factors in Computing Systems. ACM, 667-670.

[16] John Larmer and John R. Mergendoller. 2015. Gold Standard PBL: Essential Project Design Elements I Blog I Project Based Learning I BIE. Setting the Standard for Project Based Learning: A Proven Approach to Rigorous Classroom Instruction (2015).

[17] Jean Lave. 1991. Situating Learning in Communities of Practice. In Perspectives on socially shared cognition. 63-82. DOI : http://dx.doi .org/10.1037/10096-003

[18] Anne P. Massey, V. Ramesh, and Vijay Khatri. 2006. Design, development and assessment of mobile applications: The case for problem-based learning. IEEE Transactions on Education (2006). DOI : http://dx.doi.org/10.1109/TE.2006.875700

[19] Richard Noss and Celia Hoyles. 2017. Constructionism and Microworlds. In Technology Enhanced Learning: Research Themes. DOI : http://dx.doi.org/10.1007/978-3-319-02600-8_3

[20] Ofsted and Amanda Spielman. 2018. HMCI commentary: curriculum and the new education inspection framework - GOV.UK. (2018). https://www.gov.uk/government/speeches/hmcicommentary-curriculum-and-the-new-educationinspection-framework

[21] Idit Papert, Seymour and Harel and Seymour; Harel Idit Papert. 1991. Situating Constructionism.

Constructionism (1991). DOI : http://dx.doi.org/10.1111/1467-9752.00269

[22] Sean Peacock, Robert Anderson, and Clara Crivellaro. 2018. Streets for People: Engaging Children in Placemaking Through a Socio-technical Process. In Proc. of CHI (2018). DOI: http://dx.doi.org/10.1145/3173574.3173901 
[23] Farzana Rahman. 2018. Integrating Project-Based Learning in Mobile Development Course to Enhance Student Learning Experience. In Proceedings of the 19th Annual SIG Conference on Information Technology Education (SIGITE '18). ACM, New York, NY, USA, 1-6. DOI : http://dx.doi .org/10.1145/3241815.3241851

[24] Dan Richardson. 2018. GSDan/OurPlace. (2018). https://github.com/GSDan/OurPlace

[25] Dan Richardson, Clara Crivellaro, Ahmed Kharrufa, Kyle Montague, and Patrick Olivier. 2017. Exploring Public Places as Infrastructures for Civic M-Learning. In Proceedings of the 8th International Conference on Communities and Technologies - C\&T'17. ACM Press, 222-231. DOI:

http://dx.doi.org/10.1145/3083671.3083678

[26] Dan Richardson, Pradthana Jarusriboonchai, Kyle Montague, and Ahmed Kharrufa. 2018. Parklearn: creating, sharing and engaging with place-based activities for seamless mobile learning. In Proceedings of the 20th International Conference on

Human-Computer Interaction with Mobile Devices and Services - MobileHCI'18. DOI:

http://dx.doi.org/10.1145/3229434. 3229462

[27] Vidya Sarangapani, Ahmed Kharrufa, Madeline Balaam, David Leat, and Pete Wright. 2016.

Virtual.Cultural.Collaboration: Mobile Phones, Video Technology, and Cross-cultural Learning. In

Proceedings of the 18th International Conference on Human-Computer Interaction with Mobile Devices and Services (MobileHCI '16). ACM, New York, NY, USA, 341-352. DOI :

http://dx.doi.org/10.1145/2935334.2935354

[28] Vidya Sarangapani, Ahmed Kharuffa, David Leat, and Pete Wright. 2018. Creating Interactive Digital Content: A Cross-cultural Approach to Develop Critical Peer Feedback. In Proceedings of the 32nd International BCS Human Computer Interaction Conference (HCI'18). BCS Learning \& Development Ltd., Article 31, 31:1-31:11 pages. DOI :

http://dx.doi.org/10.14236/ewic/HCI2018.31
[29] Mike Sharples, Maria Aristeidou, Eloy Villasclaras-Fernández, Christothea Herodotou, and Eileen Scanlon. 2017. The Sense-it App. International Journal of Mobile and Blended Learning (2017). DOI : http://dx.doi.org/10.4018/IJMBL. 2017040102

[30] Mike Sharples, Josie Taylor, and Giasemi Vavoula. 2007. A Theory of Learning for the Mobile Age. Learning 85, 3 (2007), 221-247. DOI :

http://dx.doi.org/10.4135/9781848607859

[31] Danae Stanton, Victor Bayon, Helen Neale, Ahmed Ghali, Steve Benford, Sue Cobb, Rob Ingram, Claire O’Malley, John Wilson, and Tony Pridmore. 2001. Classroom Collaboration in the Design of Tangible Interfaces for Storytelling. In Proceedings of the SIGCHI Conference on Human Factors in Computing Systems (CHI '01). ACM, 482-489. DOI : http://dx.doi.org/10.1145/365024.365322

[32] Statistica. 2018. Children smartphone ownership 2018 । Statista. (2018).

https://www. statista.com/statistics/912577/childrensmartphone-ownership-uk/

[33] The Education Endowment Foundation. 2016. Project-Based Learning I Projects I EEF. (2016). https://educationendowmentfoundation.org.uk/projectsand-evaluation/projects/project-based-learning

[34] John Traxler and Jocelyn Wishart. 2011. Making mobile learning work: Case studies of practice. Technical Report. 46 pages. http://hdl.handle.net/2428/209909

[35] Giasemi Vavoula, Mike Sharples, Paul Rudman, Julia Meek, and Peter Lonsdale. 2009. Myartspace: Design and evaluation of support for learning with multimedia phones between classrooms and museums. Computers and Education 53, 2 (2009), 286-299. DOI : http://dx.doi .org/10.1016/j.compedu. 2009.02.007

[36] Scott Wurdinger, Jean Haar, Robert Hugg, and Jennifer Bezon. 2007. A qualitative study using project-based learning in a mainstream middle school. Improving Schools (2007). DOI : http://dx.doi.org/10.1177/1365480207078048 